\title{
5 Process Modeling using UML
}

\author{
G. ENGELS ${ }^{\dagger}$ and A. FÖRSTER ${ }^{\ddagger}$ and R. HECKEL ${ }^{\S}$ and S. THÖNE
}

University of Paderborn, Germany

\subsection{INTRODUCTION}

The Unified Modeling Language (UML) ${ }^{1}$ is a visual, object-oriented, and multi-purpose modeling language. While primarily designed for modeling software systems, it can also be used for business process modeling.

Since the early $70 \mathrm{~s}$, a large variety of languages for data and software modeling like entity-relationship diagrams [2], message sequence charts [5, 10], state-charts [9], etc. have been developed, each of them focusing on a different aspect of software structure or behavior. In the early 90s, object-oriented design approaches gained increasing attention, for instance, in the work of James Rumbaugh (Object Modeling Technique, OMT [21]), Grady Booch [1], and Ivar Jacobson [12].

The UML emerged from the intention of Rumbaugh, Booch, and Jacobson to find a common framework for their approaches and notations. Furthermore, the language was also influenced by other object-oriented approaches like, e.g., Coad/Yourdon [3]. The first version UML 1.0 [20] was released in 1997 and accepted as a standard by the Object Management Group $(O M G)^{2}$ the same year. The OMG, taking over the responsibility for the evolution of the UML from then on, is a consortium from both industry and academia also responsible for other well-known initiatives like CORBA, MDA, and XMI. OMG specifications have to undergo a sophisticated adoption process before being agreed upon as a standard by the OMG members. Since many important tool builders and influential software companies are involved in the OMG, UML has quickly been accepted by the software industry, especially since

† engels@upb.de

†alfo@upb.de

§reiko@upb.de

$\Upsilon_{\text {seb@upb.de }}$

$1_{\text {www .uml .org }}$

2 www . omg . org 
version $U M L 1.3$ in 1999. When writing this book, the current UML version is $U M L 2.0$ [18], a major revision of the language.

UML is a conglomeration of various diagram types. Therefore, the challenge is to provide a uniform framework for all these heterogeneous diagram types also accounting for relationships between them. In UML, this is solved by a common meta-model which formally defines the abstract syntax of all diagram types. The meta-model is defined with the help of the OMG MetaObject Facility (MOF) [16]. Such a declarative meta-model is an alternative to grammars usually used to define formal languages.

Besides the meta-model and a notation guide defining a concrete syntax for the meta-model elements, the UML specification also informally describes the meaning of the various meta-model elements. In the past, this informal semantics description has raised many issues about how to interpret certain details of the language. Even in the latest revision UML 2.0, there is still a number of contradictions and ambiguities to be found in the specification. At some points, the UML 2.0 specification is intentionally left incomplete, providing so-called variation points which allow tool builders and modelers to interpret the language according to their specific purposes.

This chapter provides an introduction to UML focusing especially on those parts relevant for process modeling. It covers five major aspects of process models, namely (1) actions and control flow, (2) data and object flow, (3) organizational structure, (4) interaction-centric views on business processes, and (5) system-specific process models used for process enactment. Although not every detail of the language can be presented, we intend to provide at least the most important concepts required for UML-based process models.

For discussing the various process modeling aspects, we use activity diagrams as a fundamental tool for process modeling with UML. Section 5.2 explains the control flow concepts of activity diagrams, and Section 5.3 extends the process models by integrating object flow. According to aspect (3), the modeling of underlying organizational structures is covered by Section 5.4 with the help of class and object diagrams. Section 5.5 then covers aspect (4) and deals with a different view on business processes focusing more on the interactions among involved business partners. To model such an interactioncentric view, we introduce sequence diagrams. To facilitate process enactment according to aspect (5), system-specific models should describe how to relate existing software components to the desired process activities. Thus, Section 5.6 introduces structure diagrams for describing available software systems and for specifying provided operations, which are then integrated into the considered process models. The chapter is concluded by a summary and exercises of varying degrees of difficulties.

Throughout the chapter, the different diagram types are illustrated by a running example which deals with an e-business company selling hardware products. For simplicity reasons, the company's product range is limited to monitors and computers only. It processes incoming orders by testing, assembling and shipping the demanded products. 


\subsection{MODELING CONTROL FLOW WITH ACTIVITY DIAGRAMS}

The basic building block of a process description in UML is the activity. An activity is a behavior consisting of a coordinated sequencing of actions. It is represented by an activity diagram. Activity diagrams visualize sequences of actions to be performed including control flow and data flow. This section deals with the control flow aspect of process models in UML.

\subsubsection{Basic Control Flow Constructs}

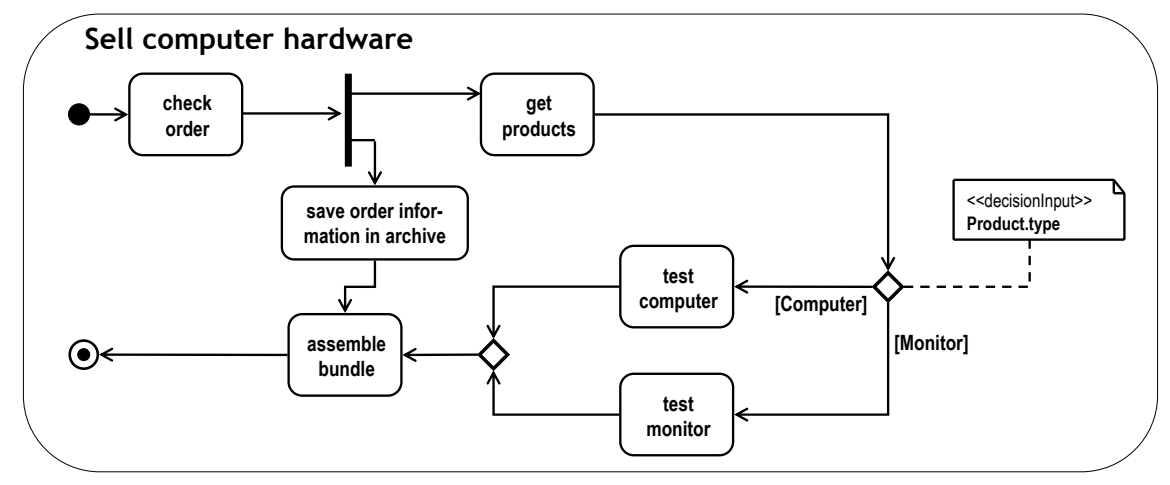

Figure 5.1 First example: computer hardware sales

Figure 5.1 shows a first small example of an activity. This activity describes a business process of our exemplary e-business company which sells computer hardware products.

The activity is visualized by a round-edged rectangle. If the activity has a name, it can be displayed in the upper left corner of the rectangle. The name of the example activity in Figure 5.1 is Sell computer hardware. Inside the activity rectangle we find a graphical notation consisting of nodes and edges that represents the activity's internal behavior. There are two kinds of nodes to model the control flow: action nodes and control nodes.

As a first step in the formulation of a business process, we need to model what tasks the process has to perform while executing. In an activity diagram, this is described by actions. An action stands for the fact that some transformation or processing in the modeled system has to be performed. Activities represent the coordinated execution of actions. Action nodes are notated as a round-edged rectangle, much like that of an activity, but smaller. Actions have a name that is displayed inside the action symbol, for instance check order or get products in our example. Actions can manipulate, test and transform data or can be a call to another activity. What has to be done when executing an 
action can be described by the name of the action such as check order. Actions can also be specified using programming language expressions such as $c:=a+b$ or formal expressions. The execution of actions takes place over a period of time.

Actions need to be coordinated. This coordination of actions within an activity is expressed by control flow edges and control nodes. The most fundamental control structure is the sequence, in which one action can start executing when another action stops executing. A simple example of a sequence of actions can be seen in Figure 5.2. The stick-arrowhead lines between the action nodes are called activity edges which specify the control flow.

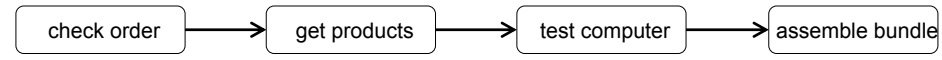

Figure 5.2 Sequence of actions

In UML 2.0, the semantics of activities is defined based on token flow. Tokens can be anonymous and undistinguishable; in that case they are called control tokens. Tokens can also reference to data objects. These tokens are called object tokens. See Section 5.3 for an introduction into the concept of object flow.

Tokens flow along the control edges thus determining the dependencies in the execution of the actions. Actions can only begin execution when tokens are available from all preceding actions along the incoming edges (step 1 in Figure 5.3). When the execution of the action starts, all input tokens are consumed and removed from the incoming control flow edges simultaneously (step 1 and 2 in Figure 5.3). After completion of the action, tokens are offered to all outgoing edges simultaneously (step 3 ).

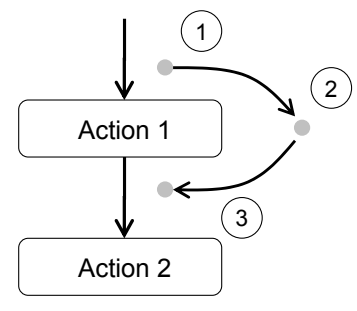

Figure 5.3 Token flow

In a control flow, actions sometimes have to be executed alternatively depending on conditions. This corresponds to the control structure often called "XOR-split" or "simple choice" (see Chapter 9), which is represented in activity diagrams by decision nodes, merge nodes and guards. The diamond symbol in Figure 5.4 represents a decision node if one edge enters the node and mul- 
tiple edges leave it. In the opposite case, if multiple edges enter the diamond symbol and one leaves it, it is a merge node which corresponds to an "XORjoin". Diamond symbols with both multiple edges entering and multiple edges leaving it are combined decision and merge nodes.

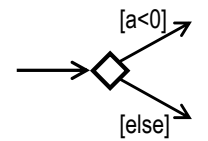

decision node with multiple outgoing edges and guards

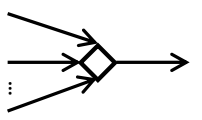

merge node

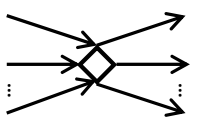

combined decision/merge node

Figure 5.4 Decision node notations

In order do describe the conditions for the choice of the alternative control flows, the edges leaving a decision node are usually annotated by guards. Guards are logical expressions that can evaluate to true or false. They can be formulated using natural language, programming language constructs or formal expressions such as mathematical logic or OCL. OCL stands for $O b$ ject Constraint Language [17], which is also developed by the OMG. It is a language for describing constraints whenever expressions over UML models are required. In an activity diagram, guards have to be enclosed in squared brackets. An edge can only be traversed if the guard attached to that edge, if any, evaluates to true.

If a guard expression becomes very lengthy, one can also attach a «decisionInput» note box to the diamond containing the text of the guard condition. This note box is connected to the decision node with a dashed line as in Figure 5.1. In the example, a product is either a computer or a monitor. As there exist two different test facilities for monitors and computers, the control flow has to be split into two different alternatives.

A special case of a guard is [else], which evaluates to true if and only if all other guards on all other edges leaving the same node evaluate to false. The use of guards is not restricted to edges leaving decision nodes. As a general rule, control edges can only be traversed if their guard condition evaluates to true.

In process models, one frequently has to model concurrent control flows. Concurrency in activity diagrams can be expressed by using fork and join nodes. They are equivalent to the concept of "AND-splits" and "AND-joins" described in Chapter 9. A thick-lined bar is a fork node if one edge enters it and multiple edges leave it as in Figure 5.5. At a fork node, the control token becomes duplicated and the control flow is broken into multiple separate control flows that execute in parallel. In order to simplify the model, one can also draw multiple outgoing edges leaving an action node (implicit fork). In our example in Figure 5.1, the action save order information in archive can be 
executed in parallel with the action get products and the product tests, as indicated by the fork node.

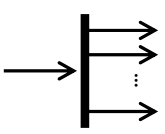

fork node

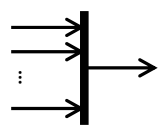

join node

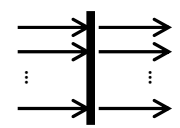

combined fork/join node

Figure 5.5 Fork/join node notations

A join node is used to combine the concurrent control flows again. It is represented by a thick-lined bar with multiple edges entering it and one edge leaving it. It synchronizes the control flows at the incoming edges since the execution is stopped until there are tokens pending along all incoming edges. A thick-lined bar with multiple incoming and outgoing edges is a combined join and fork node as depicted in Figure 5.5. Actions with multiple incoming edges represent implicit joins as the action assemble bundle in our example in Figure 5.1. Figure 5.6 shows an action with implicit fork and join.

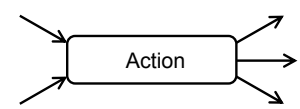

Figure 5.6 Action with multiple incoming and outgoing edges and implicit fork/join

In Figure 5.1, there are two more control nodes. A solid circle indicates an initial node which is the starting point for an activity. A solid circle surrounded by a hollow circle is the final node indicating the end of the control flow. It is possible to have more than one final node in one activity. In that case, the first final node reached stops all flows in the activity. A detailed analysis of control structures in workflow models can be found, for example, in [13].

\subsubsection{Advanced Concepts}

Pre- and postconditions In process models, it is often required to formulate assertions and conditions that need to hold locally at certain points in the control flow, at the overall beginning of an activity, or at its end.

In order to express global conditions for an activity, the activity can be constrained with pre- and postconditions. Whenever the activity starts, the precondition is validated. Whenever the activity ends, the postcondition has to be fulfilled. Both pre- and postconditions are modeler-defined constraints. They are indicated by the keywords «precondition» and «postcondition», typically in the upper part of an activity box like in Figure 5.7 a). 
Local pre- and postconditions can be attached to actions. They are displayed as note boxes containing the keywords «localPrecondition» or «localPostcondition» as in Figure $5.7 \mathrm{~b}$ ). A token can only traverse an edge when it satisfies the postconditions of the source node, the guard condition for the edge and the preconditions for the target node all at once. The constraints can be formulated in natural language, programming language expressions or any formal language like OCL, mathematical logic, etc.

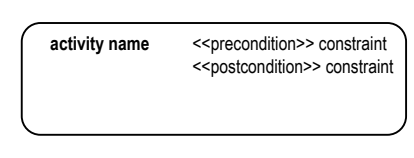

a)

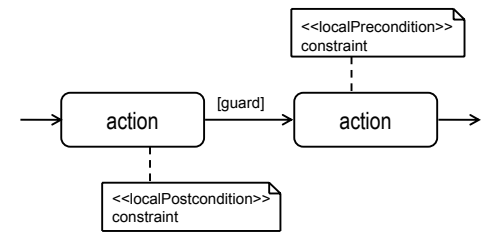

b)

Figure 5.7 Pre- and postconditions

Hierarchical process composition Business processes can easily become very complex. It is advantageous for a process description language to allow hierarchical nesting in order to reduce the complexity. Thus, actions as part of a UML activity can be calls to other activities. The nesting of activities results in a call hierarchy in which activities can be found on different levels of abstraction. An action that calls another activity is symbolized by a hierarchy fork within the action symbol (see action test computer in Figure 5.8.)

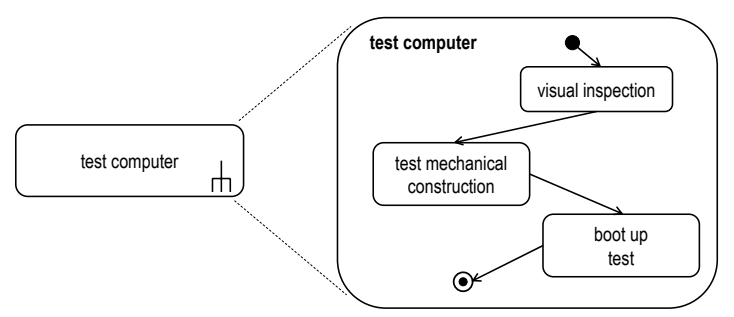

Figure 5.8 Example of an activity call

Edge weights In business processes it is sometimes necessary to describe a situation in which a defined number of objects or tokens have to accumulate at a certain point in the process before the execution can continue. In our example, one needs to collect all monitors and computers of an order before they can be bundled for shipment. With activity diagrams, it is possible to 
describe such situations. Edges can carry multiple tokens at the same time. They can also have weights which are displayed by writing $\{$ weight $=\mathrm{n}\}$ next to an edge. The weight expression by which $\mathrm{n}$ is replaced determines the number of tokens that are consumed from the source node on each traversal. The traversal of the edge is delayed until the required number of tokens is offered by the source node.

Connectors If edges cross large parts of a diagram, one can use connectors to split a control flow edge into two parts (see Figure 5.9). Connectors are circles containing a label. The label has to match uniquely with the label of one other connector.

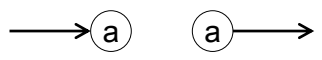

Figure 5.9 Example of an activity edge split into two parts by a labeled connector

Process interaction and signaling If the modeled system contains multiple threads of control or different activities or instances of activities running at the same time, process interaction can be required to coordinate the execution between these control flows. Process interaction can be facilitated by sending and receiving signals. In activity diagrams, there are two special nodes representing this functionality as shown in Figure 5.10: send signal action and receive signal action.

If a token reaches the send signal action, it triggers the emission of the signal. Signals can be received by receive signal actions. Corresponding send and receive actions can be determined by the signal name and optionally by a dashed line connecting sender and receiver. As soon as the signal is sent, the control token can pass on.

Receive signal actions may be included in the control flow, i.e. they have an incoming control edge. In that case, they become activated as soon as there is a token available along its incoming edge. When the incoming signal is received, the execution can continue and the control token will be passed on. Receive signal actions without incoming edges become activated as soon as the activity starts execution. After that, they can always receive signals.

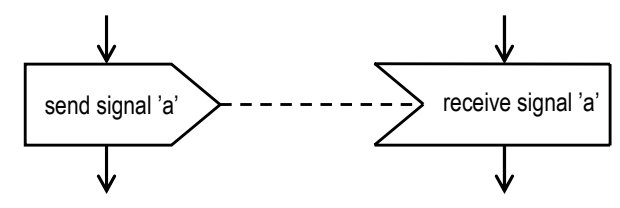

Figure 5.10 Signal send and receive actions 
Constructs to model exception handling The UML provides constructions for exception handling. A common problem is that in part of a process an exceptional condition can arise that requires actions to be performed apart from the regular workflow. This situation can be reflected in activity diagrams by introducing an interruptible activity region. Such a region contains one or more actions. It is displayed by a round-edged dashed line rectangle surrounding the actions that form the interruptible region. A lightning-bolt shaped edge called the interrupting edge leaves the interruptible region. The semantics of this construction is that if the interrupting edge is traversed, all other actions within the region are canceled and all remaining tokens within the interruptible region become abandoned. Two alternative notation options are available for the interrupting edge as shown in Figure 5.11.
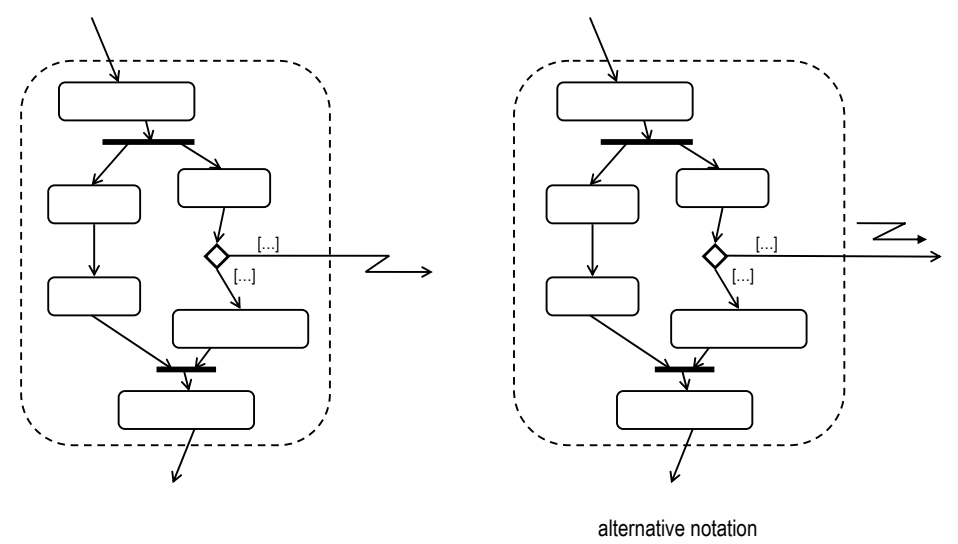

Figure 5.11 Two alternative notations for an interruptible activity region

Another exception handling situation occurs when an exceptional condition arises within one single action. For example, the action could be a mathematical division operation possibly leading to a division by zero. In activity diagrams, an exception handler can be attached to single actions as in Figure 5.12. In this case, the exception handler is a behavior that is executed whenever a predefined exception occurs while an action is being executed.

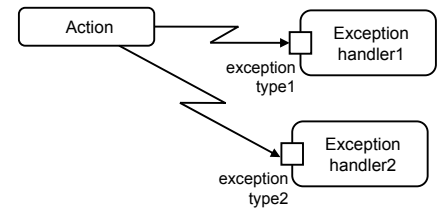

Figure 5.12 Exception handler 
Multiple exception handlers can be attached catching different types of exceptions. The execution of the exception handler substitutes the execution of the action for the time it is running. After the execution of the exception handler has terminated, the control flow is continued at the point where the execution was triggered.

The exception handler does not have own incoming and outgoing control edges since it only replaces the execution of the interrupted action. In case that an exception cannot be caught it becomes propagated to the next higher nesting or abstraction level, i.e. if the action raising the exception is part of an activity $A$ that has been called by an activity $B$, then the exception is propagated to B if it is not caught by A. If no exception handler can be found after all, the system behavior is undefined.

\subsection{MODELING OBJECTS AND OBJECT-FLOW}

All processes perform operations on physical objects, like goods that are produced out of raw materials, or logical objects, like pieces of information and data. With UML, it is possible to model the types, properties, and states of those objects as well as to integrate corresponding object flows into the activities.

For instance, consider the order handling process of our computer hardware company (see Figure 5.1), which comprises the packing of product bundles for incoming orders. This process involves two basic object types, namely hardware products and order forms. From this simple scenario, we can derive the following three requirements for modeling objects and object flow:

1. We want to model data structures, objects types (in object-oriented languages called classes) and relationship types in order to classify objects, define common properties, restrict possible relationships, and explain internal structures. For instance, we want to describe that order forms always contain a list of order items and that each item refers to a certain product type. For this purpose, we will introduce UML class diagrams.

2. We want to represent individual objects with their concrete properties and relationships. For instance, we want to describe pending orders and available products at a particular point in time. For this purpose, we will introduce $U M L$ object diagrams.

3. We want to define the dependencies between objects and actions occurring in activities, in particular input and output relationships as well as object flow dependencies. For instance, we want to describe that our packaging process requires a new order as input and how this order is processed at the different stages of the process. For this purpose, we will explain object flow concepts as part of UML activity diagrams. 


\subsubsection{Object types and instances}

Since UML is an object-oriented language, objects and their types are fundamental concepts of the language. They can be used to represent physical entities like products or persons, pieces of information like data or documents, as well as logical concepts like product types or organizations. Object types, also called classes, are defined in UML class diagrams. Objects are instances of these types, and they are represented in UML object diagrams.

Figure 5.13 summarizes the basic constructs that can be used within a class diagram. In principle, each class diagram is a graph with classes as nodes and relationships as edges. A class defines a set of common properties, also called attributes, that all instances of the class assign concrete values to. A property is defined in the second compartment of a class symbol by a property name and a property type like, e.g., String, Integer, etc.

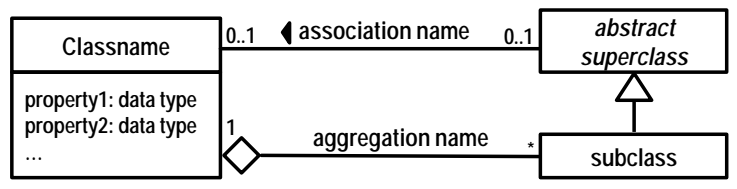

Figure 5.13 Basic class diagram constructs

Besides the classes as object types, a class diagram can contain three different kinds of relationship types (cf. Figure 5.13):

- A generalization relationship (depicted as a triangle-shaped arrow) is used to factorize common properties of different classes in a common superclass. The subclasses inherit all the properties and associations of their superclasses. If it is not intended or meaningful to create own instances of the superclass, it can be declared to be an abstract class (indicated by its name printed in italics).

- An association (depicted as a line between classes) is used to define possible links between objects. The usual form are binary associations between exactly two classes. Besides a name, an association has cardinality constraints at its ends which are given as a fixed value or as a range of lower and upper bounds (the symbol * means "unbounded"). For each association end, the cardinality constraint restricts the number of objects that can be associated to an instance of the opposite association end. A small solid arrowhead next to the association name can be used to indicate a reading direction for ambiguous association names.

- An aggregation (depicted as an association with a diamond symbol next to the container class) is a special association indicating a containment relationship. It is used to model object types which have other objects as parts. 


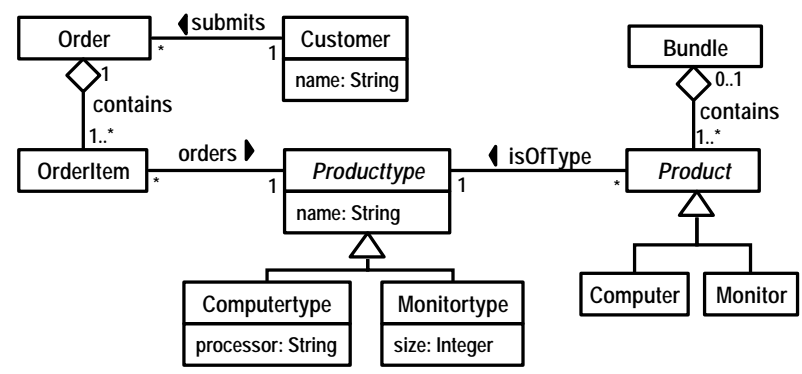

Figure 5.14 Class diagram example

Coming back to our example, consider the class diagram in Figure 5.14. It states that every Order is submitted by a Customer and that it is composed out of one or more Orderltems. The Producttype class and its subclasses Computertype and Monitortype are used to describe the product range of the company. Every Orderltem refers to a Producttype that the customer wants to order. The Product class and its subclasses Computer and Monitor are used to describe the physical products to be sold. The association isOfType between Product and Producttype is used to assign a type to every product. Both Product and Producttype are abstract classes so that only their subclasses can have instances. Due to the generalization, the subclasses inherit the isTypeOf association and the name attribute. Products can be aggregated to a Bundle.

Objects, being instances of the defined classes, have unique identifiers and concrete values for their properties. A snapshot of the objects existing at a certain point in time is modeled by a UML object diagram, as shown in Figure 5.15 for our application example. In contrast to classes, objects are depicted with underlined identifiers and type names. Objects that are parts of composite objects can be shown within the rectangle of the container object.

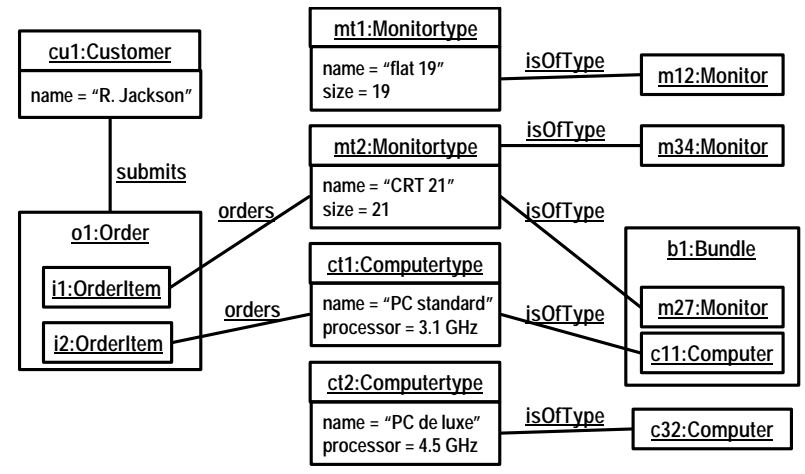

Figure 5.15 Object diagram example 


\subsubsection{Extending activities with object flows}

In Section 5.2, we introduced UML activities solely focusing on the control flow aspect. Now, we can combine the control flow with object flow.

In UML activities, we use object nodes to model the occurrence of objects at a particular point of the process. If we expect objects of a certain type only, we can type object nodes by one of the classes defined in the class diagram. Since business processes usually perform transformations on physical objects or data objects, it is often useful to add information about the current state in the object life cycle to an object node. In general, an object node is depicted as a rectangle containing the type name and, in squared brackets, the state information as shown in Figure 5.16 a).

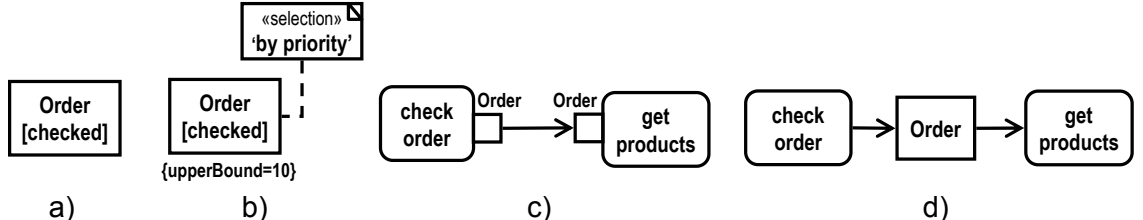

Figure 5.16 Object nodes (a,b), connected pins (c), and standalone notation (d)

In order to also capture object flow, the token flow semantics of activity diagrams is extended by object tokens. An object token behaves like a control token, but, in addition, it carries a reference to a certain object. Edges between object nodes represent flows of such object tokens. If the target object node of such an edge has a type, it can only accept tokens with objects that are instances of this type. Thus, the modeler has to consider type compatibility, and an object flow edge is only allowed if the type of the target object node is the same or a supertype of the type of the source object node.

Whenever an object token arrives at an object node it is immediately offered along outgoing edges to downstream nodes. If the node has more than one outgoing edges, they have to compete for the object token and only one of them can retrieve it. If no guard condition is given, the winning edge is determined non-deterministically. Otherwise, if we want to allow all downstream nodes to have concurrent access to the object, we can insert an explicit fork node since this causes a duplication of the object token. Then, each downstream node receives a token referring to the same object.

However, if none of the downstream nodes is ready to accept tokens, the object node can temporarily store the tokens and pass them on in the same order (FIFO, "first-in-first-out"). Instead of FIFO, one can also specify a different kind of queuing orders like LIFO ("last-in-first-out"), 'by priority', etc. by a suitable «selection» note as shown in Figure 5.16 b). Moreover, an upper bound can be given that restricts the number of tokens allowed to 
accumulate in an object node. Object tokens cannot flow into the node if that limit has already been reached.

With the help of object nodes and object flows, we can model how objects are directed through the different actions of an activity and how they are assigned to the input and output parameters of the various actions. To facilitate the latter, object nodes can also appear in the form of input pins and output pins which are directly attached to an action node. Input pins are assigned to the input parameters of the action, and output pins to its output parameters. As shown in Figure $5.16 \mathrm{c}$ ), pins are depicted as small hollow squares with their types written above the square.

An action can start execution only if all its input pins hold an object token. Then, the action consumes the tokens from its input pins and, after completion, places new object tokens on all of its output pins. Figure $5.16 \mathrm{c}$ ) shows two actions whose output and input pins are connected by an object flow edge. If the connected output and input parameters have the same name and type, the standalone notation can be used instead of the two pins as shown in Figure $5.16 \mathrm{~d}$ ).

In the following paragraphs, we show how these object flow concepts apply to our example business process of Figure 5.1, and we explain the different usages of object nodes in more detail. The resulting extended activity model is shown in Figure 5.17.

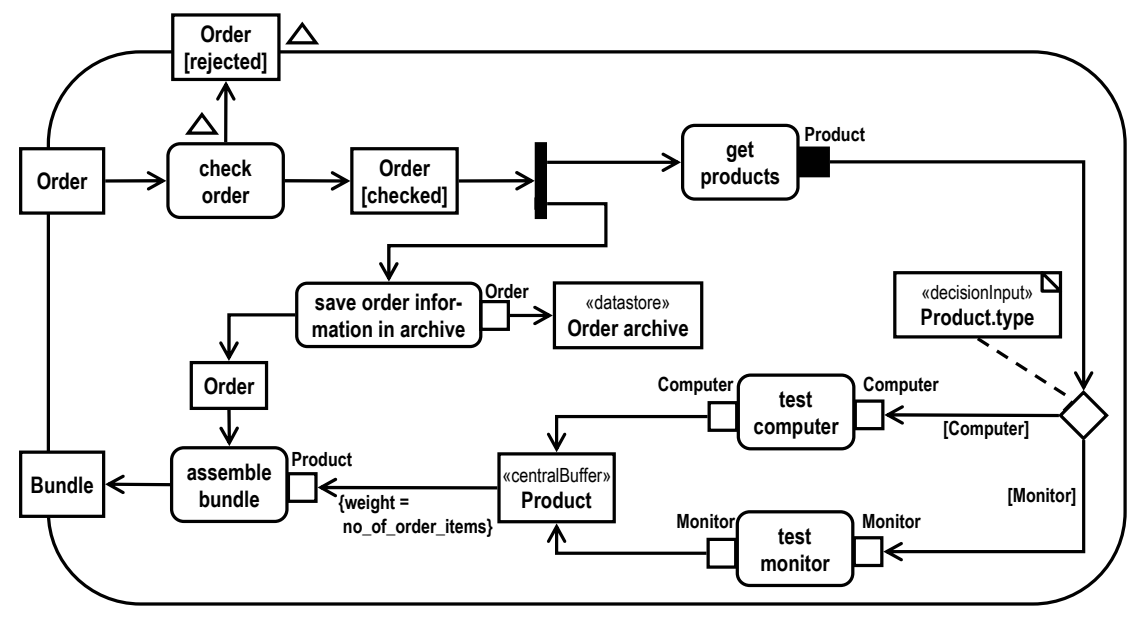

Figure 5.17 Example activity with object flow

Similarly to the individual actions, the overall activity can have input and output parameters, too. Those activity parameters are modeled with object nodes playing the role of activity parameter nodes. In our order process for instance, each arrival of an Order object places a corresponding object token at the input parameter node of the activity. From there, the token is directed 
to the first action, and the process is executed until the last action places a token with the Bundle object at the output parameter node.

The first action check order of the process validates an incoming Order object and, if successful, passes it on through its output pin. Since the downstream actions require Order objects in the state checked, too, we can use the standalone notation for object nodes here.

If the check is not successful, we want the process to terminate and to reject the invalid order. We can model this as an exception output parameter: Both actions and activities can have such output parameters that are used only when an exception occurs. As shown for check order in Figure 5.17, the output pins and output parameter nodes for exceptions are indicated by a small triangle. A token is placed there only after an abnormal termination. Otherwise, if the action or activity completes successfully, it does not place any object token there.

According to the control flow model of Figure 5.1, the downstream actions are divided into two parallel paths. Since both of them need information from the Order object, we let the fork node duplicate the object token. One copy of the token goes to the get products action and another copy to the save order information in archive action.

The get products action takes the Order object and retrieves the ordered products from the warehouse. The resulting Product objects are placed on the output pin of the action. Since this is the only output pin of the action, the first Product token placed on that pin would already cause the termination of the action. However, we want the action to continue until it has provided individual tokens for all Product objects to be retrieved.

To generalize the problem, we require special input and output pins which associate incoming and outgoing tokens to the same execution of an action. In UML activity diagrams, this is done by declaring pins as a stream (depicted by a filled square). For instance, the output pin of the get products action in Figure 5.17 is a stream. Actions with streaming output pins can continue to place tokens there while they are executing. Similarly, actions with streaming input pins can continue to accept new input tokens while a single execution of the action is running.

Coming back to our example, we want the subsequent testing actions to treat the retrieved Product object tokens separately because each product has to pass its own quality test. Consequently, the pins of the testing actions are not declared as streams again. Since different tests are required for computers and monitors, a decision node is used to direct the products to the right test action. As shown in the example, we can use information about objects and their attributes in the branching conditions.

After the quality test, the products should be collected again before they are assembled into bundles. We can model this by a central buffer node which is a special object node (labeled as «centralBuffer») that can be used to manage object flows of various incoming and outgoing edges. Central buffer nodes are not directly connected to actions but to other object nodes or pins. 
Thus, they provide additional, explicit means for queuing object tokens. In our example, the buffer type Product is compatible to both upstream types Computer and Monitor since they are subtypes according to the class diagram of Figure 5.14.

The save order information in archive action has to store statistical information about the order in an archive. If we want to model such persistent storage of data, we can use data store nodes which are a specialization of central buffer nodes (labeled as «datastore»). In contrast to central buffer nodes, a data store node keeps all tokens that enter it copying them when they are chosen to move downstream.

Eventually, the assemble bundle action packages all Product objects into a Bundle object and passes it to the output parameter node of the activity. The action must not start execution unless all ordered products have finished the quality test and are available from the central buffer. This can be guaranteed by the weight expression which delays the object flow until as many Product tokens are available as order items are contained in the Order.

\subsection{MODELING ORGANIZATIONAL STRUCTURE}

The actions included in activities that describe business processes are executed by specific persons or automated systems within a company. Companies are complex socio-technical organizations. It is necessary to link the underlying organizational structure of a company to the activities of its business processes in order to describe which actions have to be performed by which organizational entity. This corresponds to the resource and organizational perspectives of workflow modeling discussed in Chapter 3. This section describes how UML can be used to address the following key requirements for modeling organizations and resources:

1. Companies consist of a multitude of organizational entities such as persons, machines and systems. Actions, for example in an activity diagram, can be associated to any of these organizational entities. To build a coherent model of a company, all these different organizational entities should be described in one single model together with their specific properties and relationships. Examples of such relationships are leadership hierarchies, ownership and shareholder relationships, department affiliation, project group affiliation, communication structures etc. We will use UML object diagrams to model concrete organizations.

2. The organizational structures in companies usually follow typical patterns, such as hierarchically organized leadership structures, functional division of labor in departments or matrix organizations. With UML, it is possible to flexibly model the majority of these general organizational structures in such a way that concrete organizations can be treated as 
instances of these structures. General organizational structures can be modeled by UML class diagrams.

3. Finally, the control and object flow description contained in activity diagrams and the organizational view expressed in class and object diagrams have to be linked to each other because actions and activities need to be assigned to the organizational entities that are responsible for their execution. For this purpose, we will introduce the concepts of activity partitions and swim lanes in activity diagrams.

A general introduction into UML object and class diagrams has been presented in Section 5.3. In this section, we will focus on the usage of object and class diagrams for organizational modeling.

\subsubsection{Modeling organizational structures with object and class diagrams}

Figure 5.18 shows an example of an object diagram describing the concrete organization of our exemplary computer hardware sales company. Object diagrams are always instances of corresponding class diagrams. In this diagram, there are objects of three different classes:

- Objects of the class Employee for concrete persons;

- Objects of the class Department for departments; and

- Objects of the class Owner for legal persons that own an equity stake of the company.

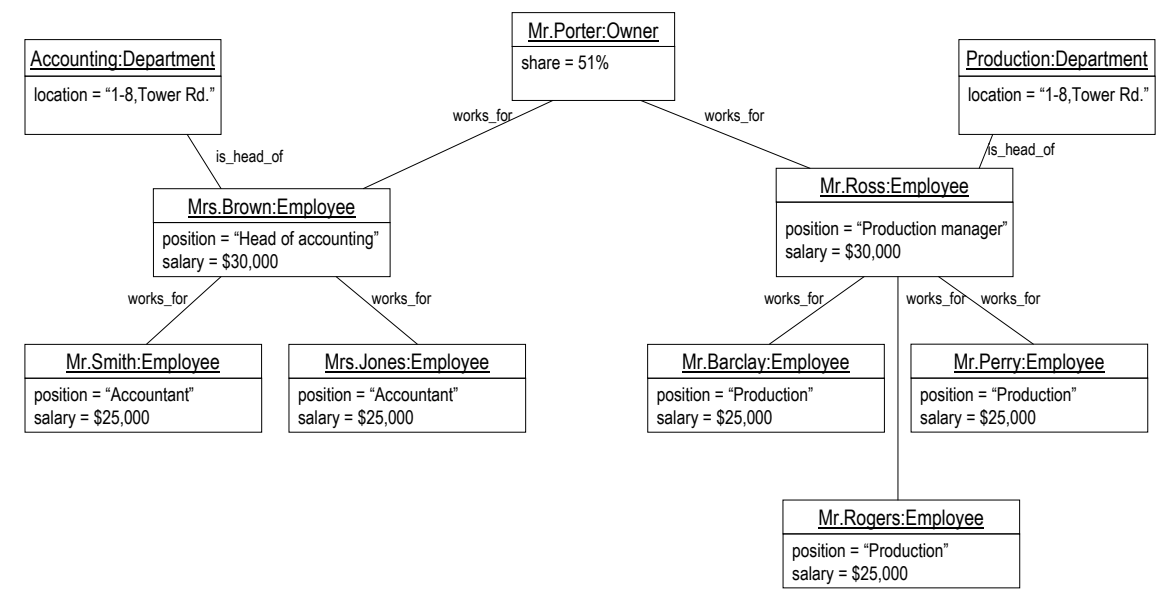

Figure 5.18 Object diagram describing a simple concrete organization 
These three classes represent three types of organizational entities in our example company. Different organizational entities can have a different set of properties. In UML, these properties are described by attributes. By associating different kinds of organizational entities to different classes, one can have different attribute sets for each kind of organizational entity. This is reflected in our example in Figure 5.18. Employees have the attributes position and salary. Departments have the attribute location and owners have the attribute share describing the equity share they own of the company. These observations lead us to the corresponding class diagram as in Figure 5.19, which describes the general organizational structure of the company. The object diagram in Figure 5.18 is an instance of this class diagram.

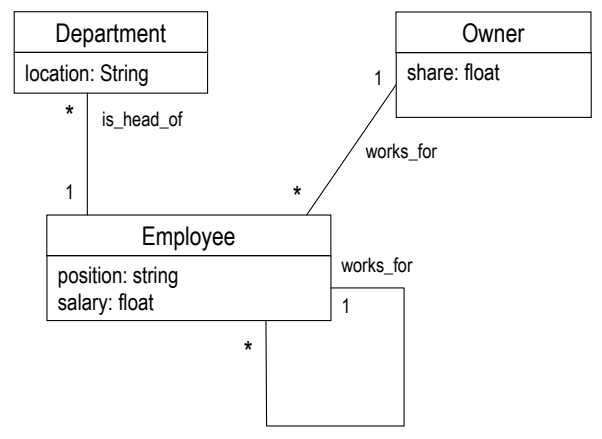

Figure 5.19 Class diagram representing a simple organizational structure

To show the full potential of organizational modeling with class diagrams, we lay down some more observations about our example company that we want to describe.

- Departments have a number of employees that work for the department. The organizational structure consists in our simplified example only of departments.

- Each department has exactly one employee or one owner as head of the department.

- The company has a board of directors that is constituted by the owners of the company.

- The owners of the company form the board of directors.

- Each employee can work for either another employee or an owner.

Figure 5.20 shows a class diagram that integrates all the observations about our example organization. Now there are classes for the organizational entities Employee, Owner, CompanyMember, BoardOfDirectors, and Department. 


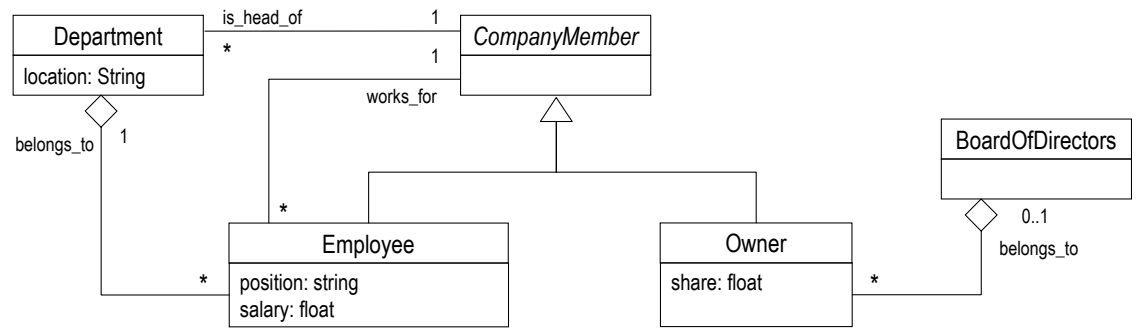

Figure 5.20 More sophisticated organizational structure

In the early version of the class diagram in Figure 5.19, Employee has two distinct associations works_for. Employees can either work for another employee or an owner. It is possible to introduce an abstract superclass CompanyMember making Employee and Owner subclasses of CompanyMember. Then the class diagram can be optimized by having only one association works_for from Employee to CompanyMember.

With the abstract superclass CompanyMember, it is also possible to model the fact that owners as well as employees can be the head of a department by changing the association is_head_of to be between Department and the new class CompanyMember. As CompanyMember is an abstract class, in an object diagram describing a concrete organization, an Employee or an Owner has to take the place of the CompanyMember. The cardinality 1 at the association is_head_of expresses that there has to be exactly one head of a department. The hierarchy of the company is built up by the departmental structure of the organization and by the association works_for.

In the class diagram of Figure 5.20, we introduce a new class representing the organizational unit BoardOfDirectors. The board of directors is built from the set of owners which is reflected by the aggregation relationship belongs_to symbolized by the association line with the diamond symbol. The Department class has an aggregation relationship to the class Employee because departments consist of employees that work for the department. The cardinality 1 expresses that every employee belongs to exactly one department.

We can now describe the complete concrete organizational structure of our example. If we add the BoardOfDirectors and the belongs_to associations to the object diagram of Figure 5.18, we yield a diagram as in Figure 5.21.

Additional remarks The structure of the class diagram in Figure 5.19 allows that in principle every employee can be subordinate to every other employee or owner, but every employee can only belong to one department. Therefore, the class diagram stipulates a hierarchical department structure.

It is also possible to describe other organizational structures than hierarchies. For example, many companies have on the one hand functional depart- 


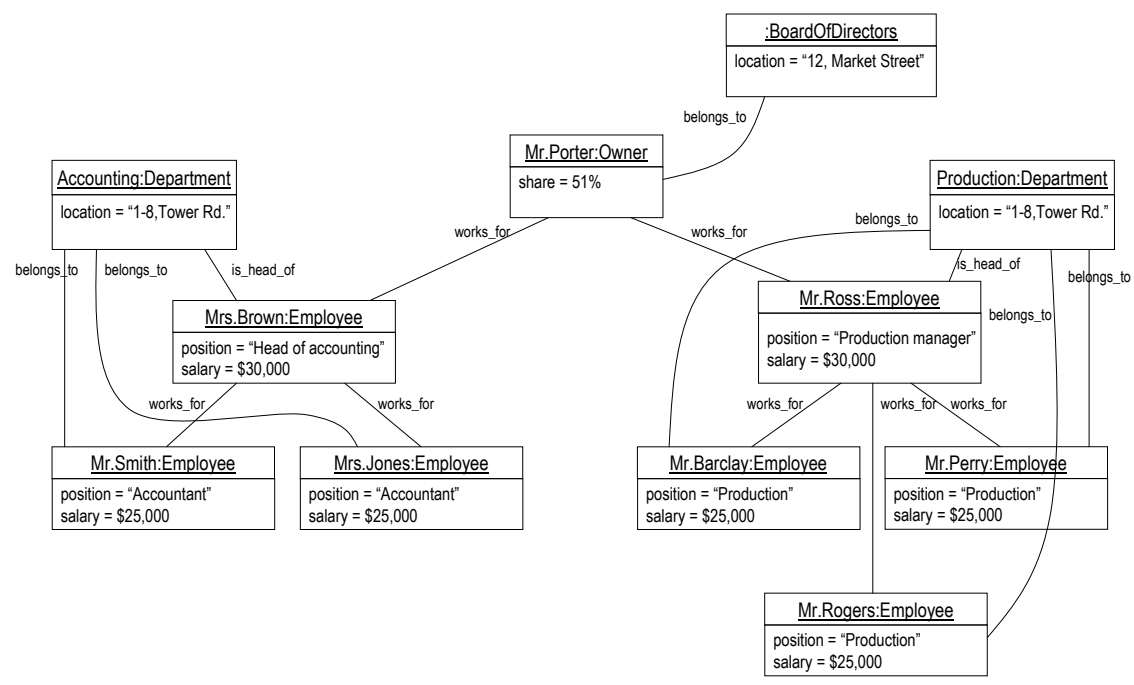

Figure 5.21 Complete object diagram for the example company

ments like "production", "accounting", "development", etc. and on the other hand departments for different product lines. This leads to a two-dimensional matrix organization. To model such an organizational structure, the cardinality 1 between employee and department has to be changed to 2. Sometimes, not every position in the matrix is staffed. For example, some employees fulfill the same function for different products. In that case, the cardinality between employee and department can be changed to 2..* for a two-dimensional matrix. Figure 5.22 shows an example of a excerpt of an object diagram for a matrix organization with two product lines for monitors and computers. Some objects and associations are left out in the diagram to account for clear arrangement. In this example, we added the department Procurement and the employee Mr.Taylor. Mr. Taylor is responsible for the procurement for both product lines, so the corresponding class diagram can be the same as in Figure 5.20 but the cardinality between employee and department has to be 2..*.

\subsubsection{Integration of organizational structures into activity diagrams}

After we have seen how organizational structures can be modeled using class diagrams and concrete organizations can be described using object diagrams, we have to connect these organizational models to the process models. In UML, this connection is done within an activity diagram using the notational elements activity partition and swim lane. 


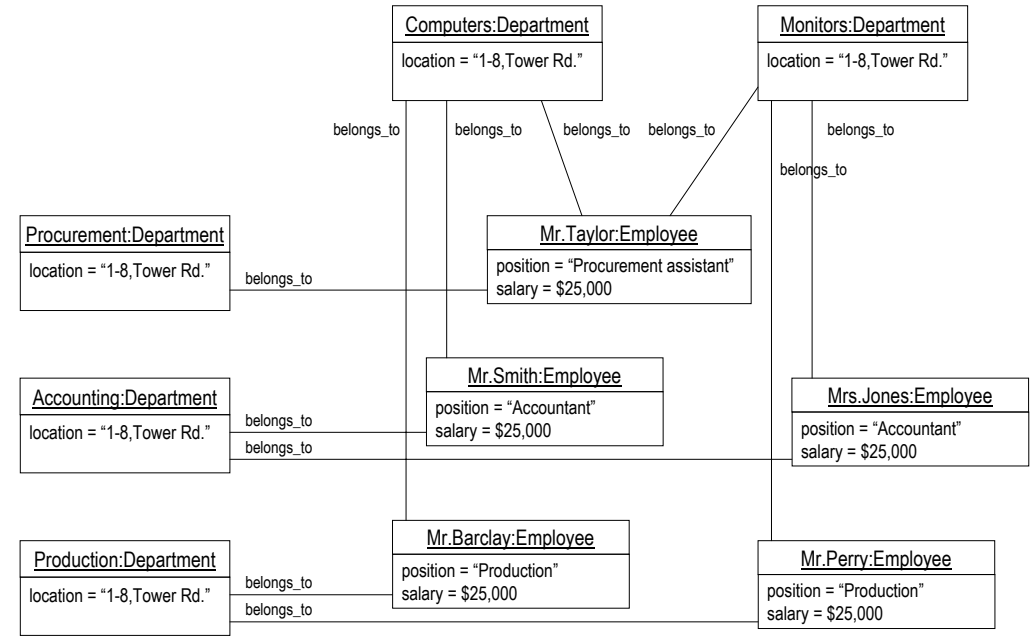

Figure 5.22 Object diagram excerpt for a matrix organization

Activity partitions divide the set of nodes within an activity into different sections. Their use is not restricted to modeling organizational units. For example, they can also be used to constrain other resources among the nodes of an activity.

Activity diagram nodes can belong to none, one or more partitions at the same time. Partitions can be divided into sub-partitions. Partitions can be visualized in two different ways. The partition name can be written in brackets over the action name within the action symbol as is Figure 5.23 a). The other possibility is the usage of swim lanes as in Figure $5.23 \mathrm{~b}$ ).

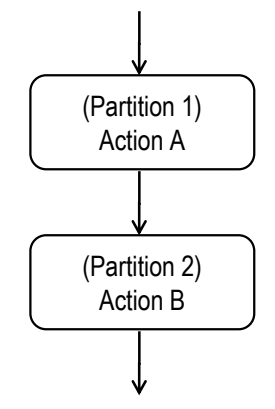

a) Partition notated on a specific activity

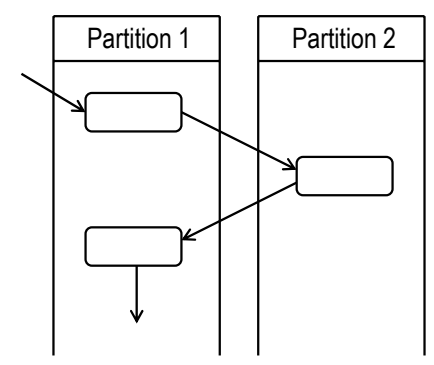

b) Swim lane notation

Figure 5.23 Actions associated to activity partitions 
Swim lanes are lines that are drawn through the activity diagram dividing it into different sections. The name of the partition is displayed on the top of the swim lane. In our case, that would be the name of the organizational unit that is responsible for execution of the actions in that partition.

With swim lanes, simple organizational structures can be reflected. In the previous section, we have introduced hierarchical and matrix organizations. Simple situations of the two organizational structures can also be displayed by swim lanes. They can be hierarchically structured as shown in Figure 5.24 a). Swim lanes can also intersect each other like in Figure $5.24 \mathrm{~b}$ ) to represent for example matrix organizations. Then the actions are associated with multiple partitions at the same time.

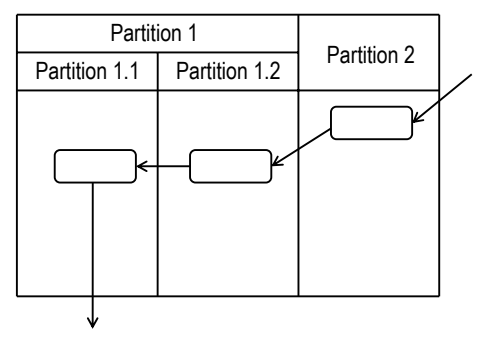

a) Hierarchy in swim lanes

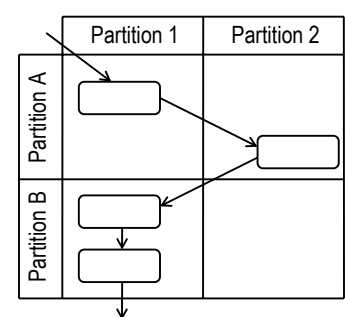

b) Multi-dimensional swim lanes

Figure 5.24 Simple organizational structures and swim lanes

The model of the organizational structure can now be integrated into the business process models of our running example. The activity depicted in Figure 5.17 contains a number of actions that have to be executed either by the accounting department or the production department. In Figure 5.25, swim lanes are included into the activity diagram to describe that the actions check order and save order information in archive are performed by the accounting department and the other actions are performed by the production department.

\subsection{MODELING BUSINESS PARTNER INTERACTIONS}

So far, we have concentrated on modeling the various dependencies between the different actions of a business process. However, a complementary view on business processes is more centered around the interactions that take place between different participants. Such interactions occur, e.g., among the employees of a certain department as well as across department and company borders. In a supply chain, for instance, the involved business partners have to interact in order to coordinate demand and supply of certain materials. 


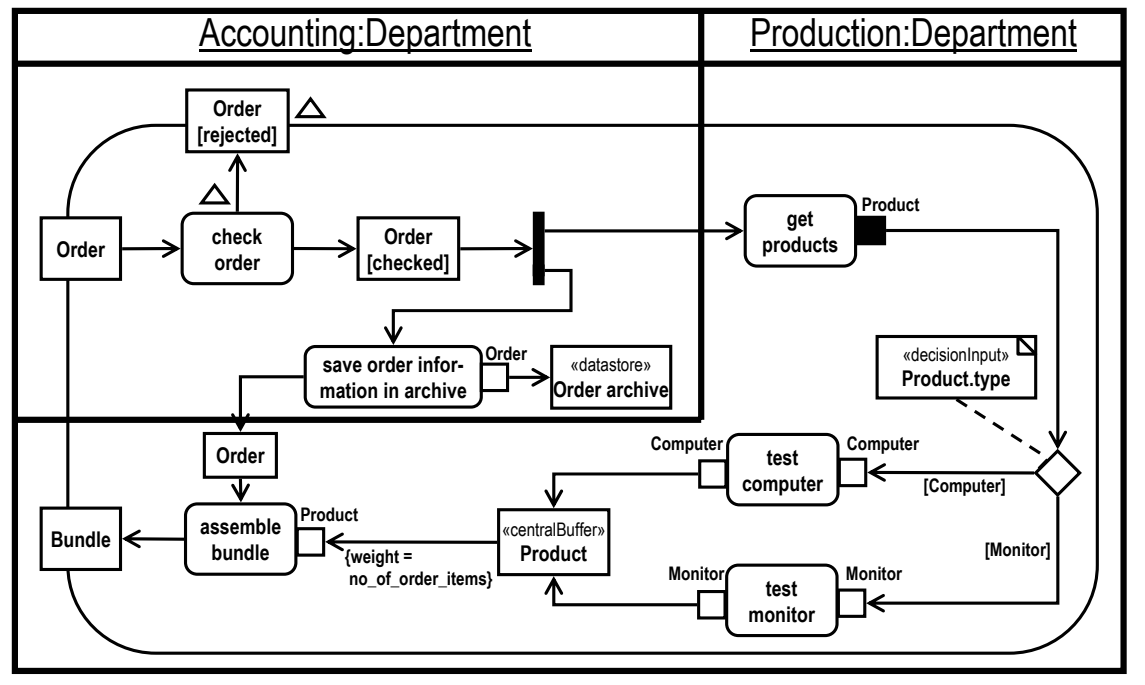

Figure 5.25 Exemplary activity with swim lane notation for the organizational entities

In such cases, the involved participants have to agree on the way they will interact. An interface process, as defined in Chapter 4, constitutes an approach to define the interactions between partners, represented by their provided endpoints. In an interface process, interactions are described from the perspective of one of the involved endpoints. As shown in Chapter 4, an interface process can be described through an activity diagram where the activities produce or consume interaction events. In some situations however, a more interaction-centric (rather than activity-centric) view on the relevant processes is more appropriate. This view allows modelers to focus on the interactions themselves, and provides a more global perspective on how multiple partners interact, as the description does not focus on the events produced or consumed by a specific participant.

For this purpose, UML provides so-called sequence diagrams. They comprise the participants involved in an interaction. Each of them has a lifeline representing its progress in time (usually from top to bottom). Arrows between the lifelines indicate the passing of a message. The sequence of arrows along the lifelines represents the order of message exchanges.

As an example, we consider the interaction of the hardware seller company with its customers, its warehouse, and a shipping service that is in charge of delivering ordered products to the customers. Figure 5.26 shows the corresponding sequence diagram.

This sequence diagram is named order interactions and comprises a Customer, the Company, its Warehouse, and the ShippingService as participants. 


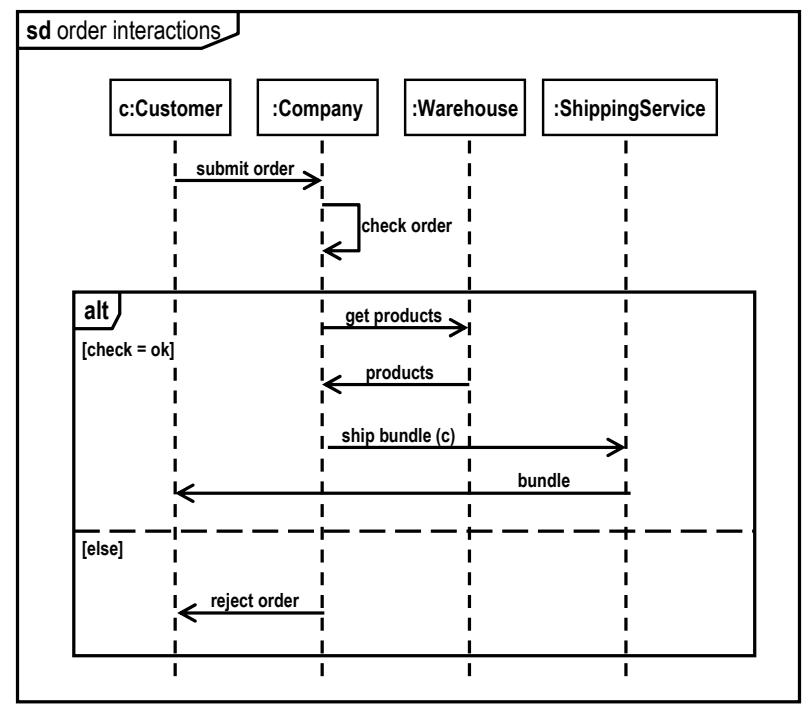

Figure 5.26 Sequence diagram for interactions related to order processing

Every participant is depicted as a rectangle which contains the name of the participant and its type. In contrast to the notation of objects in object diagrams, these names and types are not underlined because they represent a certain role rather than a concrete instance. At process enactment time, the role names have to be bound to concrete entities of the specified type. For instance, a concrete customer submits the order and a specific shipping service is selected. If the role name is not referenced later in the diagram, one can also omit it and specify just the role type.

The messages attached to the arrows represent, e.g., a request for some provided service, a response to the requester, the transmission of a certain signal, the sending of a certain return value, the transportation of some objects, and so forth. Like with action names in activities, there are different degrees of formalization possible starting from simple keywords down to operation calls with formal parameters.

In UML sequence diagrams, one distinguishes between synchronous (filled arrowhead) and asynchronous (open arrowhead) message passing. The synchronous mode means that the sender stops its activity after sending the message and waits until the corresponding response message arrives. In our example, we use only asynchronous message passing, meaning that the partners remain active after having sent a message independent of the response.

In our example, the Customer at first submits the order which is then checked by the Company. Although one should usually abstract from internal actions like check order and concentrate on external interactions in sequence 
diagrams, we can still model such internal actions as self-related messages if they have an impact on the remaining part of the interaction. In our example, this is the case because the downstream interactions are divided into two alternative interaction fragments (indicated by the keyword alt and the subdivided rectangle) which are chosen according to the outcome of the check order action. Either the order is valid and the products can be retrieved from the Warehouse and delivered by the ShippingService, or the order is not valid and rejected to the Customer.

Besides the alt operator for alternatives, sequence diagrams also provide other interaction operators that can be used in combination with interaction fragments, e.g., the loop operator indicating that a certain fragment is repeated as long as a certain condition holds, or the par operator indicating that several fragments are executed in parallel. Besides, different fragments can also be nested to model more complex interactions.

Such interaction model provides a complementary view on the business processes modeled before. In contrast to the activity diagrams, they usually hide internal actions which do not affect other participants (e.g., the testing actions of Figure 5.1 and 5.17). Nevertheless, the two different views on the business process must be consistent with each other, which means that they have to preserve the order of overlapping actions and events. For example, in both views Figure 5.1 and Figure 5.26 the check order action comes before the get products action.

\subsection{SYSTEM-SPECIFIC PROCESS MODELS}

The so far presented business process models can be used for, e.g., design, analysis, or documentation purposes. However, another purpose of process models is to support process enactment. In this case, they have to be refined into activities with atomic actions that are not further subdivided. These actions can then either be performed by humans or executed by machines and computers.

At this point, we want to focus on the latter case where processes mainly transform pieces of information and can therefore be enacted with the help of computer systems (i.e. application-to-application processes). We model the available software components of an enterprise and relate their services to the actions of our process model. Thus, we receive a refined, system-specific model that can be used for process enactment. In the terminology introduced in Chapter 4, this type of model corresponds to an integration process.

In principle, such system-specific process descriptions can be used in two ways. The first option is to feed them into a central process engine that has access to all available software components and invokes their services according to the process description (see Figure $5.27 \mathrm{a}$ ). Thus, the process engine is responsible for managing the various process instances, the control flow, and the object flow. 
The second option is to take the more local point of view of a single component which realizes a new service by using a set of services provided by other components. Then, the process model can be used to describe how the invocations of the required services are coordinated in order to realize the desired service (see Figure 5.27 b).
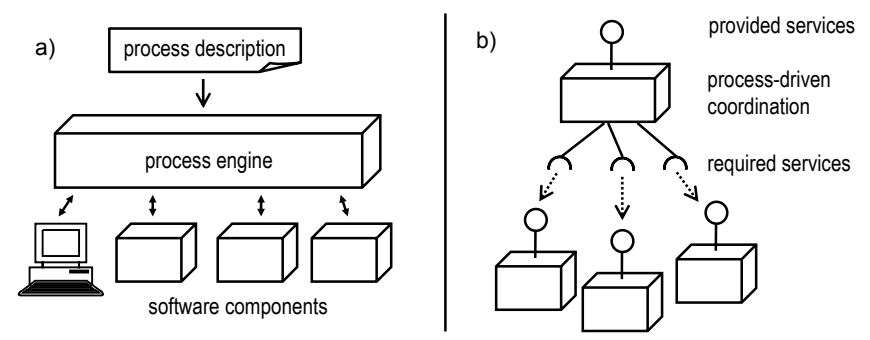

Figure 5.27 Central process engine (a) and process-driven service coordination (b)

For instance, service-oriented architectures consist of distributed software components that make use of existing third-party services in order to provide new services. Since this usually involves components of different business partners, process descriptions are needed to adjust the invocation behavior among the different partners. The Business Process Execution Language for Web Services (see Chapter 14) is a textual language for implementing such architectures in which process-driven coordination of services takes place.

As an example of system-specific models, we refine the check order action used in the order processing activity of Figure 5.17 and specify how existing services are combined to realize this action. For this purpose, we have to decompose the action into atomic subtasks like evaluating the customer's credit rating and checking the available product supplies. We assume that there are software components including, e.g., warehouse and customer management systems which provide services for these tasks. This leads to the following requirements:

1. We require a model of available systems and components which abstracts from their internal computations but specifies their provided and required services. For instance, we want to describe that there is an order management system which provides the service to check incoming orders; and, in order to do so, it requires certain warehouse and rating services. For this purpose, we will introduce UML structure diagrams and interface descriptions.

2. Having specified the provided and required services, we want to integrate them into our process models in order to coordinate their invocation. For instance, we want to describe in which way the services required by the order management system are invoked in order to realize the provided order checking service. Since inputs required by one service might 
be provided as outputs by other services, we have to consider both control and object flow dependencies. The resulting system-specific process models should serve as a basis for computer-based process execution.

UML structure diagrams provide a high-level view on existing information systems as shown in Figure 5.28. Components are depicted as boxes omitting details about their internal computations. Provided and required services, in UML called operations, are summarized into interfaces of the components. Provided interfaces are depicted as a circle connected to the providing component, required interfaces as a half-circle connected to the requiring component.

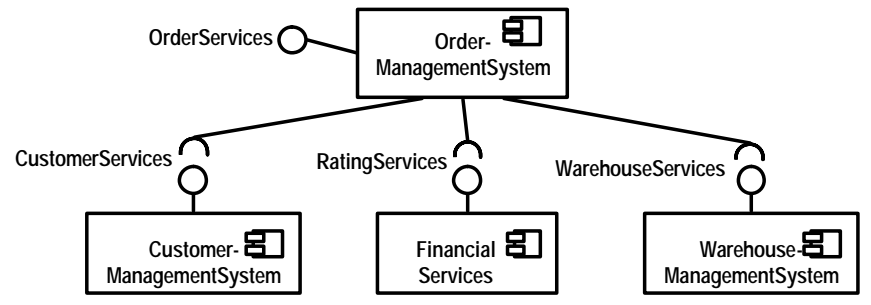

Figure 5.28 Structure diagram example

For each required interface, another component is needed which can provide a matching interface. In our case, the CustomerManagementSystem provides CustomerServices to the OrderManagementSystem, the FinancialServices component provides the RatingServices interface, and the WarehouseManagementSystem provides the WarehouseServices interface.

Interfaces are specified in a simple form of class diagram as shown in Figure 5.29. In contrast to classes used for modeling object structures, the focus is not on structural properties and relationships but on operations. An operation signature is defined in the second compartment of the interface symbol by a name and a set of input and output parameters. If there is not more than one output parameter, we can list the input parameters in parentheses and append the output parameter as return type of the operation at the end. Otherwise, we have to distinguish input and output parameters by the keywords in, out, or inout (see, e.g., the checkOrder operation of the OrderServices interface).
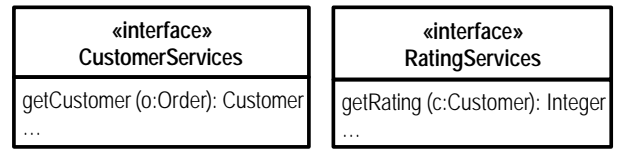

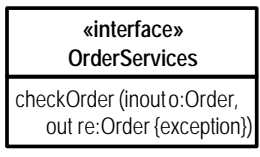

Figure 5.29 Interface specifications 
In contrast to ordinary classes, interfaces cannot be instantiated but they can only be used to indicate that a class or component either provides or requires the set of operations defined in the interface. In order to integrate the invocation of these operations into our process models, we introduce call actions for activity diagrams.

In general, call actions represent the invocation of certain behaviors defined in accompanying diagrams. In our case, we use them to call operations of component interfaces as shown in the system-specific checkOrder activity (Figure 5.30). In contrast to ordinary action nodes, the node symbol contains the exact name of the operation to be called. Below the operation name, the name of the interface or component type providing the operation is added in brackets. All input and output parameters defined in the operation signature are transformed into input and output pins of the action node. Thus, when defining the control flow between the call action nodes, one has to consider object flow dependencies that arise from the operation's input and output behavior.

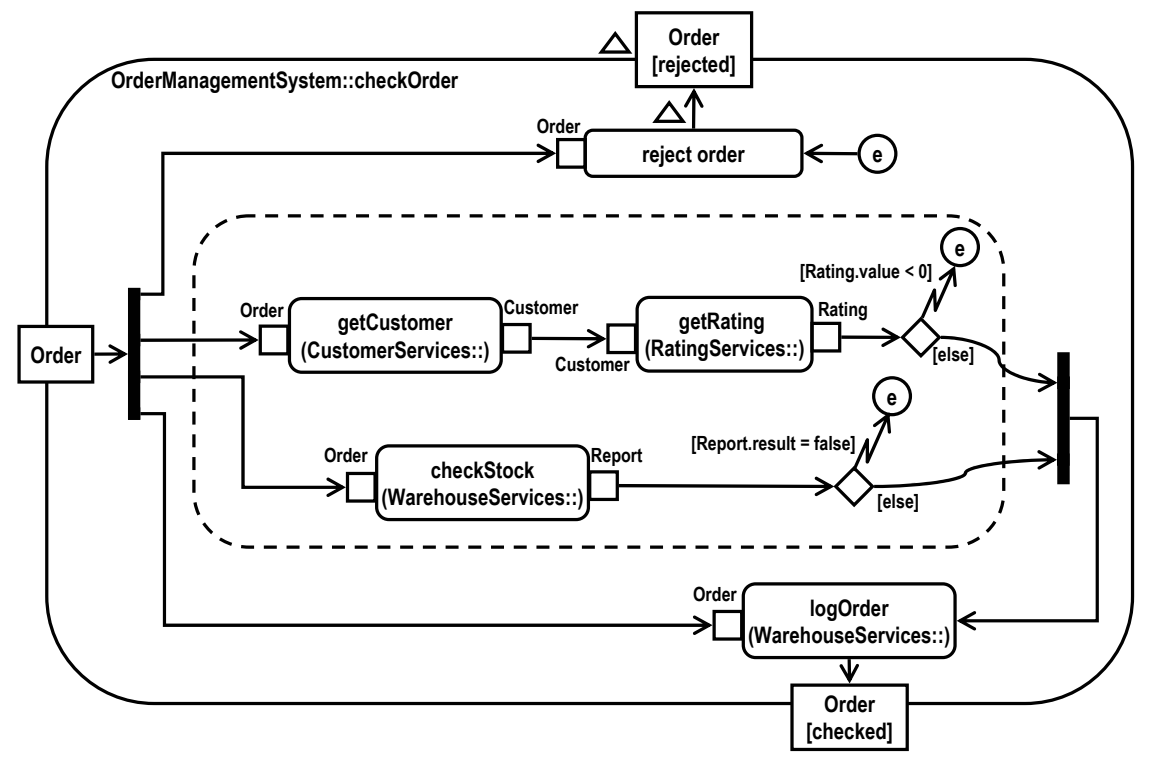

Figure 5.30 System-specific activity diagram for the checkOrder service

Since, according to the interface description in Figure 5.29, the checkOrder operation has an inout parameter of type Order, the activity gets a corresponding input parameter node, too. From there, incoming Order objects are passed on to the action nodes of the activity until they are eventually placed on the output parameter node shown at the bottom of Figure 5.30. 
If any of the involved checks returns a negative result, then the Order is rejected and placed at the second output parameter node (shown at the top of Figure 5.30), which is an exception as indicated by the small triangle. Note that exactly this arrangement of parameter nodes is required if we want to use the activity as an refinement of the check order action of Figure 5.17.

The activity involves two checks that can be performed in parallel: Firstly, the customer's credit rating should have a positive value, and secondly, the available product supplies of the warehouse should be sufficient to satisfy the demand. Since the getRating operation of the RatingServices interface requires a Customer object as input parameter (see Figure 5.29), we have to insert an action calling the getCustomer operation first. This CustomerManagementSystem operation retrieves the corresponding Customer object from an associated database, which is then passed on to the getRating operation.

The two parallel action flows for order checking are enclosed in an interruptible region so that any negative result prevents further effort and directly leads to the reject order action throwing an exception. However, if both parallel checks are successful, the interruptible region is left, and the logOrder operation of the WarehouseServices interface is invoked to update the product information stored in the WarehouseManagementSystem. Eventually, the checked Order is returned as output to the superior process.

As revealed by this example, system-specific process models refine actions and activities of more abstract, business-level process models. Given a mapping of the interfaces to real components with physical addresses (also called deployment description), such system-specific process models can be used for process enactment and coordination of the involved software components. For related work about using activity diagrams in order to integrate applications and software components, the interested reader is referred to [6] and [22].

\subsection{SUMMARY}

Modeling processes requires the description of a number of different perspectives of the process $[11,4]$. We have covered five major perspectives of process modeling with UML diagrams. This includes the description of actions and control flow, data and object flow, organizational structure, interaction-centric views, and application integration through system-specific, refined process models for process enactment. Table 5.7 summarizes which UML diagrams we have employed to describe these process modeling perspectives respectively.

For further studies of the UML, the interested reader can find detailed insights into the language concepts in the book by Pender [19]. How to apply the UML for developing information systems from requirements analysis to system design is described, for instance, in the work by Maciaszek [14].

There are strong efforts to further increase the usability of UML for process modeling. The recent revision UML 2.0 has already improved, among others, the suitability of activity diagrams. In order to further extend the language 


\begin{tabular}{l|c|c|c|c|c} 
& $\begin{array}{c}\text { activity } \\
\text { diagram }\end{array}$ & $\begin{array}{c}\text { class } \\
\text { diagram }\end{array}$ & $\begin{array}{c}\text { object } \\
\text { diagram }\end{array}$ & $\begin{array}{c}\text { sequence } \\
\text { diagram }\end{array}$ & $\begin{array}{c}\text { structure } \\
\text { diagram }\end{array}$ \\
\hline actions and control flow & $\mathrm{X}$ & & & & \\
\hline data and object flow & $\mathrm{X}$ & $\mathrm{X}$ & $\mathrm{X}$ & & \\
\hline organizational structure & $(\mathrm{X})$ & $\mathrm{X}$ & $\mathrm{X}$ & & \\
\hline interaction-centric view & & & & $\mathrm{X}$ & \\
\hline system-specific models & $\mathrm{X}$ & $(\mathrm{X})$ & & & $\mathrm{X}$
\end{tabular}

Table 5.1 Overview of the different UML diagrams

according to business process modeling requirements, one can also use the built-in extension mechanisms of UML. These extensibility features allow designers to adapt certain parts of the language to their domain-specific needs while still remaining within the framework of the UML meta-model. For this purpose, so-called stereotypes can be defined which describe semantic extensions as well as syntactical modifications of dedicated meta-model elements. A set of related stereotype definitions forms a UML profile.

Work in progress includes the development of a specialized business process definition profile by the OMG [15]. The objective is to allow groups using a variety of process models, including UML activity diagrams and other process modeling notations, to map to a common meta-model and thus facilitate communication among themselves.

Among others, there are efforts to increase the support for collaborating business processes, business process patterns, runtime implications of process definitions, resource assignments, access control etc. The extensibility feature of UML will facilitate the efforts to further develop extensions of the UML for business process modeling in order to make it even more powerful and user-friendly.

\subsection{EXERCISES}

1. Consider the test computer and test monitor actions in Figure 5.17 and model the case when such a product test fails. For this purpose, you could, e.g., add output pins returning a test report. If the report reveals a negative test result, a substitute product has to be retrieved from the warehouse and the test has to be redone.

2. As a preparation for modeling the internals of the testing actions, extend the class diagram of Figure 5.14 as follows: A checklist is associated to each product type. Every such list contains a set of items that describe 
the properties to be checked for the associated product type. Each item has a property name and a reference value as attributes.

3. Now, refine the test computer action of Figure 5.17 into an activity showing the internals of the action. Model the input and output parameter nodes of the activity according to the pins of the corresponding action node. The activity should contain an archive for all the checklists for the various product types. Whenever a new computer object arrives, the right checklist has to be selected from the archive. You can then freely design your own control and object flow to realize the testing activity.

4. Extend the interaction model of Figure 5.26 with the company's house bank as additional business partner. After ordered products have been delivered to the customer, the company sends a bill to the customer containing a reference to the bank. Then, the customer can transmit the payment to the company's bank account. In a second step, try to model that the delivery of the products and the payment can also happen in parallel.

5. Consider the object diagram for the example company in Figure 5.21 and the matrix organization excerpt in Figure 5.22. How would a complete object diagram of the company look like if you combined the two existing diagrams?

6. In the matrix organization in Figure 5.22, we use the organizational entity Department both for the functional entities of the company like procurement, accounting etc. and for the product oriented entities like monitor and computer. Devise an organizational structure that contains departments and product lines as two distinct organizational entities. Extend the organizational model developed in Section 5.4 with the necessary additional classes. What additional associations have to be defined? How would the object diagram in Figure 5.22 be affected?

7. In Figure 5.21, Mr. Ross is an employee. Now assume that Mr. Ross is not only an employee but also an owner of the company at the same time. How could this be modeled in the class diagram (hint: consider multiple inheritance)? How would the object diagram in Figure 5.21 be affected? 



\section{References}

1. G. Booch. Object-Oriented Analysis and Design with Applications. Addison Wesley, 2nd edition, 1994.

2. P. Chen. The entity-relationship model - toward a unified view of data. ACM Transactions on Database Systems, Vol. 1, No. 1:9-36, 1976.

3. P. Coad and E. Yourdon. Object-Oriented Analysis. Yourdon Press, 2nd edition, 1991.

4. B. Curtis, M. I. Kellner, and J. Over. Process modeling. Communications of the ACM, 35(9), 1992.

5. W. Damm and D. Harel. LSCs: Breathing life into message sequence charts. Formal Methods in System Design, 19(1):45-80, 2001.

6. R. Depke, G. Engels, M. Langham, B. Lütkemeier, and S. Thöne. Processoriented, consistent integration of software components. IEEE Proc. of the 26th Int. Computer Software and Applications Conference (COMPSAC), pages $13-18,2002$.

7. G. Engels, R. Heckel, and J. M. Küster. The consistency workbench: A tool for consistency management in UML-based development. Proceedings UML 2003 - The Unified Modeling Language, Springer LNCS 2863:356$359,2003$.

8. A. Förster. Quality ensuring development of software processes. European Workshop on Software Process Technology (EWSPT), Springer LNCS 2786:62-73, 2003.

9. D. Harel. Statecharts: A visual formalism for complex systems. Science of Computer Programming, 8(3):231-274, June 1987.

10. ITU-TS, Geneva. ITU-TS Recommendation Z.120: Message Sequence Chart (MSC), 1996.

11. St. Jablonski and C. Bussler. Workflow Management: Modeling Concepts, Architecture and Implementation. International Thomson Computer Press, London, 1996. 
12. I. Jacobson, M. Christerson, P. Jonsson, and G. Overgaard. ObjectOriented Software Engineering - A use case driven approach. Addison Wesley, 1992.

13. B. Kiepuszewski, A.H.M. ter Hofstede, and W.M.P. van der Aalst. Fundamentals of Control Flow in Workflows. Acta Informatica, 39(3):143-209, 2003.

14. L. A. Maciaszek. Requirements Analysis and System Design: Developing Information Systems with UML. Addison Wesley, 2001.

15. Object Management Group. Business Process Definition Metamodel RFP. http://www.omg. org/docs/bei/03-01-06.pdf.

16. Object Management Group. Meta-Object Facility (MOF) Specification, Version 1.4. http://www.omg.org/cgi-bin/doc?formal/2002-04-03.

17. Object Management Group. UML 2.0 OCL 2nd revised submission. http: //www .omg.org/cgi-bin/doc?ad/2003-01-07.

18. Object Management Group. UML 2.0 Superstructure Final Adopted specification. http://www.omg.org/cgi-bin/doc?ptc/2003-08-02.

19. T. Pender. UML Bible. Wiley Publishing, 2003.

20. J. Rumbaugh, G. Booch, and I. Jacobson. Unified Modeling Language, Notation Guide, Version 1.0. Rational Software Corporation, Santa Clara, 1997.

21. J. E. Rumbaugh, M. Blaha, W. J. Premerlani, F. Eddy, and W. Lorensen. Object-Oriented Modeling and Design. Prentice Hall, 1990.

22. S. Thöne, R. Depke, and G. Engels. Process-oriented, flexible composition of web services with UML. Proc. of the Int. Workshop on Conceptual Modeling Approaches for e-Business (eCOMO 2002), Springer LNCS 2784:390-401, 2002. 